\title{
Managing Rush Hour Congestion with Lane Reversal and Tradable Credits
}

\author{
Qing $\mathrm{Li}^{1,2}$ and Ziyou Gao ${ }^{1}$ \\ ${ }^{1}$ School of Traffic and Transportation, Beijing Jiaotong University, Beijing 100044, China \\ ${ }^{2}$ Department of Statistics, College of Mathematics and Systems Science, Shandong University of Science and Technology, \\ Qingdao 266590, China
}

Correspondence should be addressed to Qing Li; i_liqing@163.com

Received 11 April 2014; Revised 21 June 2014; Accepted 1 July 2014; Published 4 August 2014

Academic Editor: X. Zhang

Copyright (C) 2014 Q. Li and Z. Gao. This is an open access article distributed under the Creative Commons Attribution License, which permits unrestricted use, distribution, and reproduction in any medium, provided the original work is properly cited.

\begin{abstract}
Within the morning and evening rush hour, the two-way road flows are always unbalanced in opposite directions. In order to make full advantage of the existing lanes, the two-way road lane has to be reallocated to play the best role in managing congestion. On the other hand, an effective tradable credit scheme can help to reduce the traffic demand and improve fairness for all travelers. So as to alleviate the commute congestion in urban transportation network, a discrete bilevel programming model is established in this paper. In the bilevel model, the government at the upper level reallocates lanes on the two-way road to minimize the total system cost. The traveler at the lower level chooses the optimal route on the basis of both travel time and credit charging for the lanes involved. A numerical experiment is conducted to examine the efficiency of the proposed method.
\end{abstract}

\section{Introduction}

In many big cities, a large number of people commute from residential areas to their workplaces in the central business district $(\mathrm{CBD})$ in the morning. It leads to the phenomenon that the lanes are congested in the direction from residential areas to CBD while the other lanes in the opposite direction are free. But in the evening, all of that will be reversed. In order to adjust the asymmetric amounts of traffic flow in the two directions of one road section, the lane reversal is employed as a traffic control technique. It is an effective way to make full use of existing road resource and can increase traffic supply.

Lane reversal has been used for many years in managing road congestion. In recent years, lane reversal has been introduced in Chinese big cities, such as Beijing, to relieve morning and evening commute congestion. In the last year, Chaoyang Road, a big thoroughfare on the city's east side, has been tested as the first lane reversal in Beijing to allow traffic to travel in either direction depending on certain conditions. According to the practice, the lane reversal can help to alleviate traffic congestion to some extent.
The implement of lane reversal is a network design problem (NDP). A plenty of research works are focused on the effectiveness, feasibility, and safety of implementing lane reversal [1-5]. The development of techniques, applications, and the engineering practices of lane reversal also attracts much attention [6]. In practice, improper use of lane reversal may lead to a worse condition of the whole road network. Congestion on the road with lane reversal may be relieved, but other parts of the network will be more congested. Therefore, the lane reversal must be put into use on the basis of adjustment of the whole urban road network [7, 8]. A lot of research on NDP is concerned with uncertain demand [9-12]. For simplicity, the commute traffic demand is assumed to be fixed demand. Apparently the number of reallocated lanes is integer. As a result, the implement of lane reversal is a discrete network design problem (DNDP). In essence, the lane reversal contributes to increasing road supply.

In addition to increasing supply, another efficient way to manage congestion is reducing traffic demand. It is widely acknowledged that congestion pricing is helpful in traffic demand management, but the equity debates confined its adoption all over the world. Without taking into account 
commuters' income level, trip purposes, and valuation of time, congestion pricing scheme might increase individuals' travel costs. Though it can improve the system performance, it often has to face public resistance.

According to the latest research on congestion control, tradable credit scheme is proved to be a fairer, more effective, and more practical congestion management scheme. It should be noticed that Yang and Wang first explored a tradable credit scheme in a general transportation network equilibrium context with homogeneous and heterogeneous travelers, respectively $[13,14]$. Under their credit scheme, the social planner is assumed to develop an initial distribution of the credits to all eligible travelers and link-specific charges to travelers on that link. Credits can be traded freely among travelers. It is the competitive market rather than the planner that can determine the price of the credit. An optimization model subject to a total credit consumption constraint is formulated to find the equilibrated credit price. With an appropriate distribution of credits among travelers and correct selection of link-specific rates, the results of the traditional congestion pricing scheme can be duplicated. Furthermore, the combined tradable credit scheme is shown to be both system-optimal and a Pareto-improvement in a revenueneutral manner. The same context of research has been done on the tradable credits scheme employed in managing rush hour travel choice and bottleneck congestion [15-18].

In this paper, a discrete bilevel programming model is constructed for managing rush hour congestion caused by underutilization of the existing road resource. The proposed model employs tradable credit scheme and lane reversal for increasing traffic supply and decreasing traffic demand, respectively. At the upper level, the government chooses optimal lanes to be reallocated to minimize the total system costs. Taking into account the generalized travel cost including travel time and link-specific charges for using the links, the travelers at the lower level will choose the optimal route to minimize it.

This paper is organized as follows. In Section 2, a discrete bilevel programming model is established with lane reversal and tradable credit scheme. A chaotic algorithm is employed to solve the proposed model. In Section 3, numerical experiments and analysis results are illustrated with a basic network. In Section 4, conclusion of this paper is presented.

\section{Discrete Bilevel Programming Model with Lane Reversal and Tradable Credits Scheme}

Consider a two-way network $G=(N, A)$ with a set $N$ of nodes and a set $A$ of directed links. Link in one direction of a road section is denoted by $a \in A$ and link in the opposite direction is $a^{\prime} \in A$. Let $W$ and $R_{w}$ denote the set of O-D pairs and the set of all routes between an O-D pair $w \in W$. The travel demand for each O-D pair $w \in W$, denoted by $d_{w}$, $d_{w}>0$, is given and fixed.

To all directed links in the road network, $n_{a}$ and $n_{a^{\prime}}$ denote the number of lanes on link $a \in A$ and $a^{\prime} \in A$. For simplicity, let $n_{a}=n_{a^{\prime}}$ before lane reversal. The capacity of each lane on link $a \in A$ is assumed to be equal to unity, which is denoted by $c_{a}$. That is to say, the traffic capacity of link $a \in A$ is $n_{a} \cdot c_{a}$ before lane reversal. After lane reversal, $u_{a}$ is used to denote the number of lanes in the opposite direction on link $a^{\prime} \in A$ to be added to link $a \in A$. If $u_{a}>0$, it means that $u_{a}$ lanes on the opposite direction will add to link $a \in A$ and its capacity is equal to $\left(n_{a}+u_{a}\right) \cdot c_{a}$. On the other hand, if $u_{a}<0$, it means that $u_{a}$ lanes on link $a \in A$ will be added to link $a^{\prime} \in A$ on the opposite direction. Relative to other means for network capacity enhancement, the investment of lane reversal can be ignored.

For simplicity, consider a separable link travel cost function $t_{a}\left(v_{a}, u_{a}\right)$, which is assumed to be nonnegative, continuously differentiable, convex, and monotonically increasing with respect to the amount of aggregate traffic flow $v_{a}$ on link $a \in A$ and $u_{a}$, the number of lanes to be reallocated. In general, $\partial t_{a}\left(v_{a}, y_{a}\right) / \partial y_{a}$ is assumed to be continuous too. Assume also that travelers are homogeneous, which means that they have the same value of time (VOT).

Tradable credit scheme is characterized by its initial distribution and the charging scheme. To minimize complexity, the initial distribution schemes considered here will be O-D specific for a given and fixed demand $d_{w} . k=\left\{k_{w}\right\}$ is used to denote the credit distribution scheme. Here $k_{w}$ is the amount of credit distributed to each traveler over the O-D pair $w \in W$. Let $K$ denote the total amount of credits for all links which is predetermined, and obviously $K=\sum_{w \in W} k_{w} d_{w}$. The credits are month specific so as no one can benefit from the trade of credit. Analysis here is restricted to link-specific credit charging. Let $\kappa=\left\{\kappa_{a}, a \in A\right\}$ denote the charging scheme, where $\kappa_{a}$ is the credit charge for using link $a \in A$. Then $(K, \kappa)$ will be used to represent a credit charging scheme $\kappa$ with a total number of credits $K$ for all links.

Let $f_{r}^{w}$ denote the traffic flow on route $r \in R_{w}$ between OD pair $w \in W . f$ is a path flow vector $f=\left(f_{r}^{w}, r \in R_{w}, w \in\right.$ $W$ ), and $\Omega_{f}$ represents the set of feasible path flow patterns defined as follows:

$$
\Omega_{f}=\left\{f \mid f_{r}^{w} \geq 0, \sum_{r \in R_{w}} f_{r}^{w}=d_{w}, r \in R_{w}, w \in W\right\} .
$$

Let $v=\left(v_{a}, a \in A\right)$ denote the link flow vector, and $\Omega_{f}$ represents the set of feasible link flow patterns defined as follows:

$$
\Omega_{v}=\left\{v \mid v_{a}=\sum_{w \in W} \sum_{r \in R_{w}} f_{r}^{w} \delta_{a, r}, f \in \Omega_{f}, a \in A\right\}
$$

and $\delta_{a, r}=1$ if route $r$ uses link $a$ and 0 otherwise.

It has been proved that not all $(K, \kappa)$ can guarantee the existent feasible network flow patterns. The amount of credits might not be big enough for supporting all travelers going through the network even if all of them choose the least-credit paths. In order to ensure the existence of feasible network flow patterns, the feasible set of credit schemes denoted by $\Psi$ as follows:

$$
\Psi=\left\{(K, \kappa) \mid \exists f \in \Omega_{f} \text { such that } \sum_{a \in A} v_{a} \kappa_{a} \leq K, v \in \Omega_{v}\right\}
$$

$\Psi$ is assumed to be nonempty. 
The following bilevel programming model is to minimize the sum of total system costs, while the travelers choose the optimal route for minimizing the generalized travel costs including both travel time and link-specific credit charges for using the links. The model can be described by

$$
\text { (BLP) } \min _{U} \mathrm{SC}=\sum_{a \in A} v_{a} t_{a}\left(v_{a}, u_{a}\right)
$$

subject to

$$
\begin{gathered}
u_{a} \in\left\{-n_{a},-\left(n_{a}-1\right), \ldots,-1,0,1, \ldots, n_{a}-1, n_{a}\right\}, \quad a \in A \\
u_{a}+u_{a^{\prime}}=0, \quad a \in A, a^{\prime} \in A,
\end{gathered}
$$

where $V=\left(v_{a}\left(u_{a}\right)\right)$ is the solution of the next problem

$$
\min _{V} \sum_{a \in A} \int_{0}^{v_{a}\left(u_{a}\right)} t_{a}\left(\theta, u_{a}\right) d \theta
$$

subject to

$$
\begin{gathered}
\sum_{a \in A} v_{a} \kappa_{a} \leq K, \\
\sum_{r \in R_{w}} f_{r}^{w}=d_{w}, \quad r \in R_{w}, w \in W, \\
v_{a}=\sum_{w \in W} \sum_{r \in R_{w}} f_{r}^{w} \delta_{a, r}, \quad a \in A, \\
f_{r}^{w} \geq 0, \quad \forall w \in W, \quad r \in R_{w} .
\end{gathered}
$$

The model is a mixed integer nonlinear programming program. The decision variable at upper level is integer while variable at lower level is real number. Chaos algorithm here is adopted to solve the proposed model [7]. The steps of the algorithm are as follows.

Step 1. Assume chaos variable is denote by random number $y_{a}^{0} \in[0,1], a \in A$. Let the initial optimal solution $u_{a}^{0}=0$, $a \in A, \mathrm{SC}^{0}=+\infty$. Check set is denoted by $\Phi$, and it is null set. Counter $m=1$.

Step 2. Chaos variable $y_{a}^{m}$ is generated by the following equation:

$$
y_{a}^{m}=4 y_{a}^{m-1}\left(1-y_{a}^{m-1}\right), \quad a \in A .
$$

Step 3. Carrier can be produced by the following equation:

$$
\widetilde{u}_{a}^{m}=-n_{a}-\varepsilon_{1}+\left(3 n_{a}+\varepsilon_{2}\right) y_{a}^{m}, \quad a \in A,
$$

where $\varepsilon_{1}$ and $\varepsilon_{2}$ are all small enough positive numbers, with $\varepsilon_{1}<\varepsilon_{2}$. Let $u_{a}^{m}$ be equal to rounding $\widetilde{u}_{a}^{m}, a \in A$. If $u_{a}^{m} \notin \Phi$, $a \in A$, add $u_{a}^{m}$ to $\Phi$; then turn to Step 4; otherwise, turn to Step 2.

Step 4. For given $u_{a}^{m}, a \in A$, solve the users equilibrium (UE) assignment model at the lower levels (6)-(7). The solution is the link flow vector $v^{m}=\left(v_{a}^{m}, a \in A\right)$ at UE.

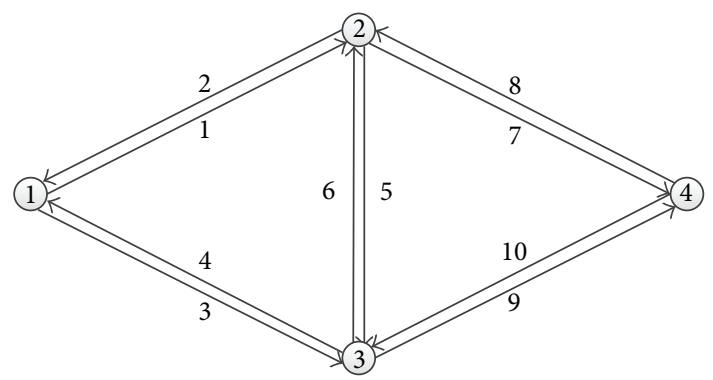

FIgURE 1: Original structure of the test network before lane reversal.

TABLE 1: Input data for the test network.

\begin{tabular}{lllcl}
\hline & \multicolumn{4}{l}{$t_{a}\left(v_{a}, u_{a}\right)=t_{a}^{0}\left(1+0.15\left(v_{a} / C_{a}\right)^{4}\right)$} \\
Link $a$ & $t_{a}^{0}$ & $C_{a}$ & $n_{a}$ & $c_{a}$ \\
\hline 1 and 2 & 4.0 & 40 & 2 & 20 \\
3 and 4 & 6.0 & 40 & 2 & 20 \\
5 and 6 & 2.0 & 60 & 3 & 20 \\
7 and 8 & 5.0 & 40 & 2 & 20 \\
9 and 10 & 3.0 & 40 & 2 & 20 \\
\hline
\end{tabular}

TABLE 2: Transportation condition before lane reversal and tradable credit scheme.

\begin{tabular}{lcc}
\hline Link $a$ & Link flow $v_{a}$ & Link utilization rate $\chi_{a}=v_{a} / C_{a}$ \\
\hline 1 and 2 & 52.5322 and 31.5193 & 1.3133 and 0.7880 \\
3 and 4 & 47.4678 and 28.4807 & 1.1867 and 0.7120 \\
5 and 6 & 15.7200 and 13.4320 & 0.2620 and 0.2239 \\
7 and 8 & 46.8122 and 28.0873 & 1.1703 and 0.7022 \\
9 and 10 & 53.1878 and 31.9127 & 1.3297 and 0.7978 \\
\hline System cost & \multicolumn{3}{c}{$\mathrm{SC}=1.8240 \times 10^{3}$}
\end{tabular}

Step 5. Solve the model at the upper level and get $S C^{k}$. If $\mathrm{SC}^{m}<\mathrm{SC}^{0}$, let SC $\mathrm{SC}^{0}, u_{a}^{0}=u_{a}^{m}, a \in A$.

Step 6. If the termination condition is met, output the optimal solution $u_{a}^{0}, a \in A$; otherwise, turn to Step 2 .

\section{Numerical Experiments}

In this paper, a basic two-way road network, as shown in Figure 1, is employed to validate the efficiency of the proposed model. The network consists of 4 nodes, 10 links, and 2 O-D pairs. One O-D pair is from node 1 to node 4 and the other is from node 4 to node 1 . In the morning rush hour, the traffic demand of the two O-D pairs is 100 and 60 in an hour.

The link cost function $t_{a}\left(v_{a}, u_{a}\right)$ used here is classical BPR function. Initial capacity of link $a \in A$, which has $n_{a}$ lanes before lane reversal and each lane's capacity is $c_{a}$, is denoted by $C_{a}=n_{a} c_{a}$. The number of $C_{a}, c_{a}, n_{a}$, and the link free flow travel time $t_{a}^{0}, a \in A$, are all illustrated in Table 1 .

The traffic flows under UE before lane reversal can be calculated and are shown in Table 2. It shows that the twoway road traffic flows are unbalanced in opposite directions. A parameter $\chi_{a}=v_{a} / C_{a}$ is introduced here to estimate the 
TABLE 3: Results after lane reversal.

\begin{tabular}{lcccc}
\hline & & Lane reversal & & \\
$u_{1}$ and $u_{2}$ & $u_{3}$ and $u_{4}$ & $u_{5}$ and $u_{6}$ & $u_{7}$ and $u_{8}$ & $u_{9}$ and $u_{10}$ \\
\hline 0 and 0 & 1 and -1 & 0 and 0 & 1 and -1 & 0 and 0 \\
\hline
\end{tabular}

TABLE 4: Transportation condition after lane reversal.

\begin{tabular}{lcc}
\hline Link $a$ & Link flow $x_{a}$ & Link utilization ratio $\chi_{a}=v_{a} / K_{a}$ \\
\hline 1 and 2 & 48.9004 and 41.3251 & 1.2225 and 1.0331 \\
3 and 4 & 51.0996 and 18.6749 & 0.8517 and 0.9337 \\
5 and 6 & 00.0000 and 22.9740 & 0.0000 and 0.3829 \\
7 and 8 & 48.9004 and 18.3510 & 0.8150 and 0.9176 \\
9 and 10 & 51.0996 and 41.6490 & 1.2775 and 1.0412 \\
\hline \multicolumn{3}{l}{ System cost } \\
\hline
\end{tabular}

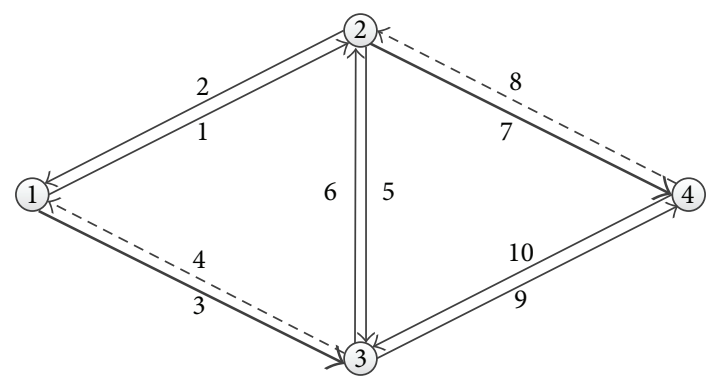

Figure 2: The structure of the test network after lane reversal.

utilization ratio of link $a \in A$. As is shown Table 2, the utilization ratios of links $1,3,7$, and 9 are bigger than 1 . That means that these links have been overused. Otherwise, the utilization ratios of the links in opposite directions are far smaller than them. It can be derived from Table 2 that the utilization ratio of link 2 is slightly bigger than half of that of link 1 . And the same happens between link 10 and link 9.

Solve the models (4)-(7) with the use of the algorithm in Section 2. The amounts of credit charging for using links are set as follows: $\kappa_{1}=\kappa_{5}=1, \kappa_{8}=2$, and $\kappa_{a}=0$ for other links $a \in A$. The parameter $\theta=0.05$ transfers money into equivalent time value. The strategy of the lane reversal and the system cost can be obtained as shown in Table 3 .

It is shown in Table 3 that one lane of link 4 and one lane of link 8 have been reversed in their directions and added to links 3 and link 7 respectively. Other lanes of other links do not have any changes. It can be found with the system cost illustrated in Table 4 that the scheme of lane reversal and tradable credits is efficient for managing rush hour congestion.

The transportation condition after lane reversal is shown in Figure 2. The lanes of links 3 and 4 and links 7 and 8 have been adjusted. The traffic flows after lane reversal can be calculated and are shown in Table 4. From the utilization ratio it can be concluded that most of the links have been made full use of. And the system cost is lower than that before lane reversal.

\section{Conclusions}

With the development of economics and city scales, the road becomes more and more congested in morning and evening rush hour. In order to achieve better effect in solving the congestion problem, traffic demand and increasing road supply are all considered at the same time in this paper. A bilevel programming model is proposed to deal with the two-way road unbalance usage problem. In order to make full advantage of the existing lanes, the two-way road lanes have to be reallocated to play the best role in managing congestion. An effective tradable credit scheme is also employed to help to alleviate the commute congestion with lane reversal in urban transportation network. The models and the algorithm are demonstrated with the basic two-way road network example.

In the future research, the heterogeneous users should be considered. Users with different job and income may have different value of time, so it will be helpful for transportation planning to simulate the real situation.

\section{Conflict of Interests}

The authors declare that there is no conflict of interests regarding the publication of this paper.

\section{Acknowledgments}

This study was jointly supported by the National Basic Research Program of China (2012CB725401) and the National Natural Science Foundation of China (71271205 and 71322102).

\section{References}

[1] J. Hemphill and V. H. Surti, "A feasibility study of a facility for a Denver street corridor," Transportation Research Record, vol. 514, pp. 29-32, 1974.

[2] R. J. Caudill and N. M. Kuo, "Development of an interactive planning model for contraflow lane evaluation," Transportation Research Record, vol. 906, pp. 47-54, 1983.

[3] Y. Jiang and L. Bao, "Study on setting of variable lanes near intersection between one-way and two-way traffic," Journal of Shanghai Jiaotong University, vol. 45, no. 10, pp. 1562-1566, 2011.

[4] F. Ni and Z. Liu, "The optimal location of variable message sign," Information and Control, vol. 32, no. 5, pp. 395-398, 2003.

[5] X. Gong and S. Kang, "Study and application of traffic direction changing algorithm for urban tide traffic situation," Journal of Transportation Systerns Engineering and Information Technology, vol. 6, no. 6, pp. 33-40, 2006.

[6] P. B. Wolshon and L. Lambert, Convertible Roadways and Lanes: A Synthesis of Highway Practice, NCHRP Synthesis 340, National Cooperative Highway Research Program, Transportation Research Board, Washington, DC, USA, 2004. 
[7] H. Zhang and Z. Gao, "Optimaization approach for traffic road network design problem," Chinese Journal of Management Science, vol. 15, no. 2, pp. 86-91, 2007.

[8] X. Liang, C. Hu, C. Ma, and B. Mao, "Empirical study on variable lanes design of chaoyang north street in Beijing," in Proceedings of the 30th Chinese Control Conference (CCC '11), pp. 5527-5531, July 2011.

[9] H. Shao, W. H. K. Lam, and M. L. Tam, "A reliability-based stochastic traffic assignment model for network with multiple user classes under uncertainty in demand," Networks and Spatial Economics, vol. 6, no. 3-4, pp. 173-204, 2006.

[10] H. Shao, Q. Tian, X. Yuan, and G. Wang, "Risk-taking path choice behaviors under ATIS in transportation networks with demand uncertainty," Journal of Southeast University, vol. 24, pp. 43-48, 2008.

[11] H. Shao, W. H. K. Lam, Q. Meng, and M. L. Tam, "Travel time reliability-based traffic assignment problem," Journal of Mangement Sciences in China, vol. 12, no. 5, pp. 27-35, 2009.

[12] H. Shao, W. H. K. Lam, A. Sumalee, and A. Chen, "Journey time estimator for assessment of road network performance under demand uncertainty," Transportation Research C: Emerging Technologies, vol. 35, pp. 244-262, 2013.

[13] H. Yang and X. Wang, "Managing network mobility with tradable credits," Transportation Research B: Methodological, vol. 45, no. 3, pp. 580-594, 2011.

[14] X. Wang, H. Yang, D. Zhu, and C. Li, "Tradable travel credits for congestion management with heterogeneous users," Transportation Research E: Logistics and Transportation Review, vol. 48, no. 2, pp. 426-437, 2012.

[15] Y. M. Nie and Y. Yin, "Managing rush hour travel choices with tradable credit scheme," Transportation Research B: Methodological, vol. 50, pp. 1-19, 2013.

[16] F. Xiao, Z. Qian, and H. M. Zhang, "Managing bottleneck congestion with tradable credits," Transportation Research B: Methodological, vol. 56, pp. 1-14, 2013.

[17] L. Tian, H. Yang, and H. Huang, "Tradable credit schemes for managing bottleneck congestion and modal split with heterogeneous users," Transportation Research E, vol. 54, pp. 113,2013 .

[18] G. Wang, Z. Gao, M. Xu, and H. Sun, "Models and a relaxation algorithm for continuous network design problem with a tradable credit scheme and equity constraints," Computers \& Operations Research, vol. 41, pp. 252-261, 2014. 


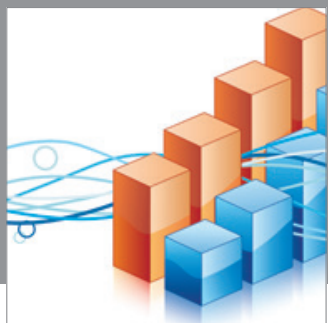

Advances in

Operations Research

mansans

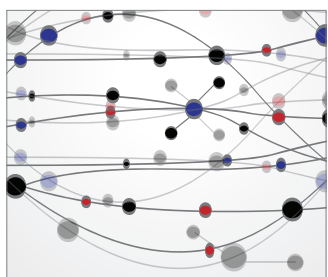

The Scientific World Journal
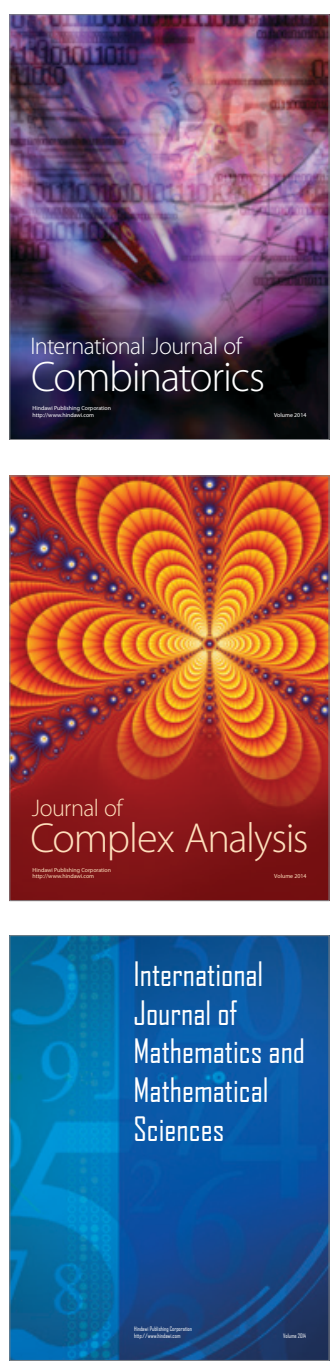
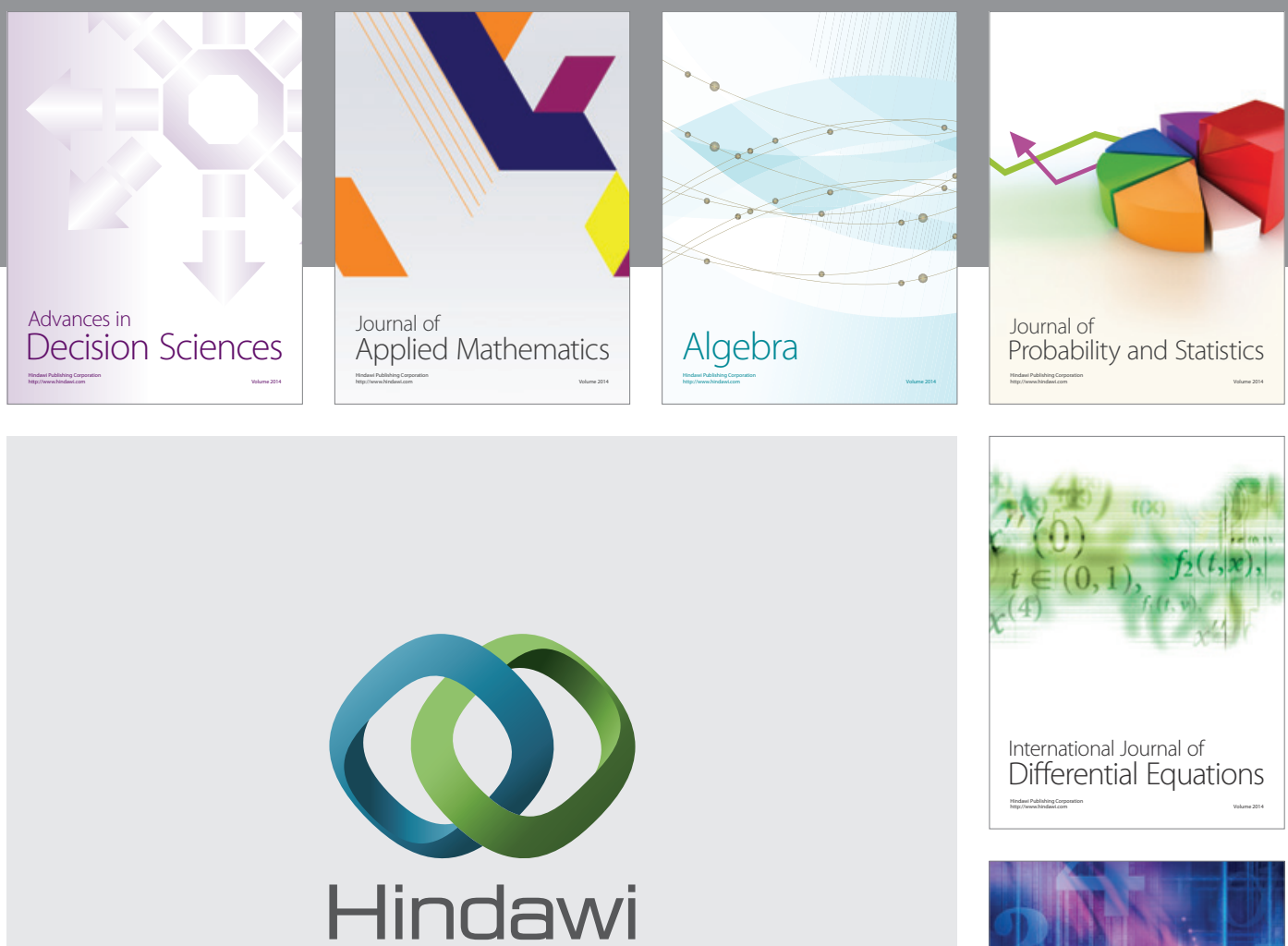

Submit your manuscripts at http://www.hindawi.com
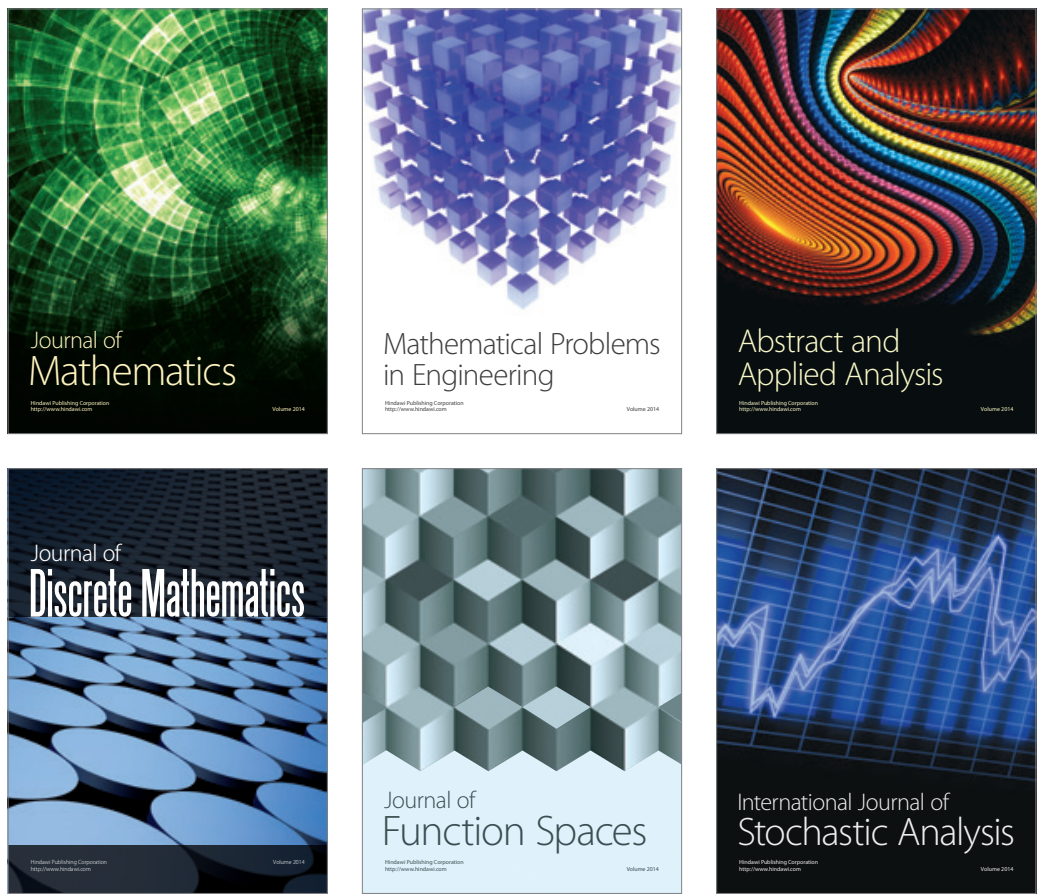

Journal of

Function Spaces

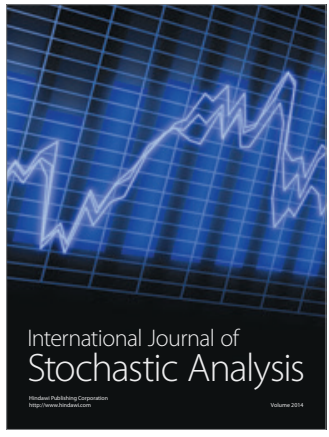

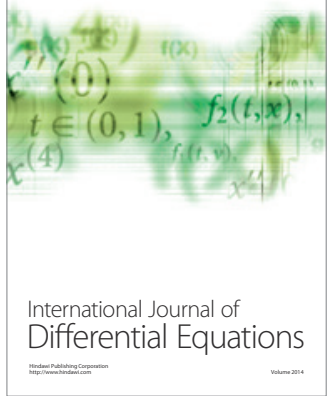
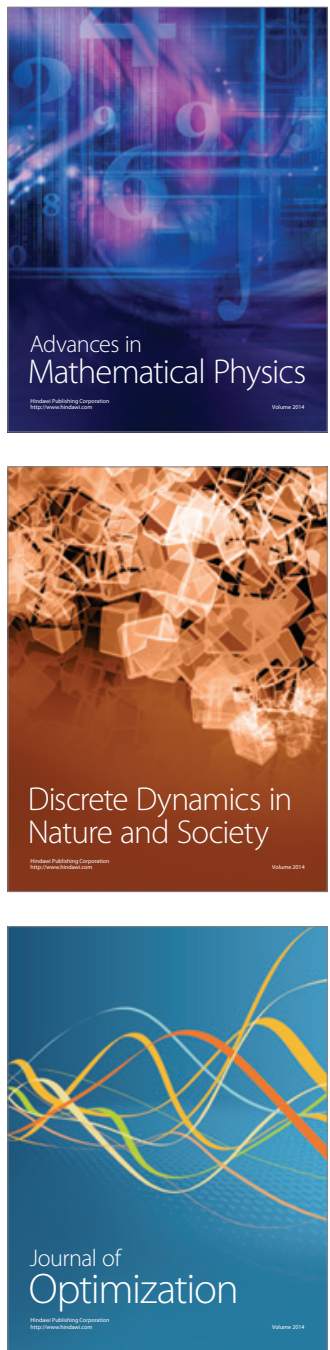